\title{
Design and Implementation of a Class D Power Amplifier for a Transmitter in a Sonar System
}

\author{
SONG Hong Yan ${ }^{1, a}$, WEN Ming ${ }^{1}$, CHEN Guang $\mathrm{Da}^{1}$ and DENG Hong Chao ${ }^{1}$ \\ ${ }^{1}$ Shanghai Marine Electronic Equipment Research Institute, Shanghai, 201108, China \\ agabe513@sina.com
}

Keywords: Class D Power Amplifier; Transmitter; Sonar System

\begin{abstract}
The power amplifier operating in a low frequency with high output power is always the hot issue in the sonar transmitter system in underwater acoustic field. This paper provides a Class D power amplifier for a transmitter in a sonar system. We first describe the basic principles of power amplifiers. Then we give the details when designing and fabricating a switched Class D power amplifier. Lastly the test results are offered. As a result of measurement, the power amplifier can operate in a center frequency of $100 \mathrm{kHz}$ with a bandwidth of $5 \mathrm{kHz}$, a $760 \mathrm{~W}$ output power.
\end{abstract}

\section{Introduction}

Sonar, an acronym for SOund Navigation And Ranging, is a technique that uses sound propagation usually underwater to navigate, communicate with or detect objects on or under the surface of water. Generally, two types of technology share the same name "sonar": the passive sonar and the active one. The passive sonar is only used to detect the sound made by vessels, frogmen, submarines and so on. When an active sonar working, it emits some pulses of sounds at first, and then waits to listen for the echoes reflected from underwater targets. Nowadays sonar equipments have a wide range of use whether in civilian areas or in military field [1].

Transmitter, as an important part of a sonar system, transports high power signals to transducers underwater. When receiving the electrical signals from power amplifier with high power, transducers convert them to strong acoustic signals over a distance. Power amplifier is the most important component of the transmitter system. In sonar application, the power amplifier means the audio power amplifier. The audio power amplifier is an electronic amplifier that amplifies low-power audio signals (signals composed primarily of frequencies between $20 \mathrm{~Hz}-20000 \mathrm{~Hz}$ ) to a high power level suitable for driving transducers. As can be seen, the power level of the power amplifier's output signal can affect the detection range of the sonar equipment directly.

In this paper, we designed a switched Class D power amplifier based on a transmitter platform using in a sonar system. The core specifications such as operating frequency, bandwidth and output power were all tested.

\section{Principles of Class D Power Amplifier}

"Power Amplifier", often abbreviated as "PA", as the name suggests, is mainly used to amplify the small input signals to a high power level for the load in the next stage. Usually, operating frequency, bandwidth, output power and other specifications are used to measure the performance of a PA. According to different position of quiescent operating point setting, the amplifiers can be generally classified as: Class A, Class B, Class AB, Class C, Class D, Class E and some other types. Class A, Class B, Class AB and Class $C$ amplifiers are linear ones. Transistors in these amplifiers operate approximately in linear region. Otherwise, the Class D PA is completely a different new type of power amplifier, and can be called "the digital audio amplifier".

Transistors in Class D PA operate in switch mode using for amplifying input signals. In the ideal state, the resistance is approximately zero when transistors are turned on; the resistance is nearly infinite when transistors are shut off [2]. The basic schematic diagram of Class D PA is shown in Figure 1. 


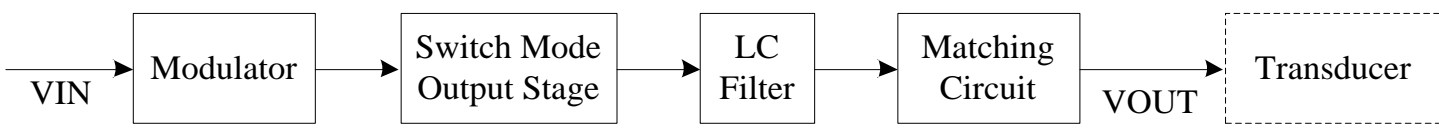

Fig.1 Basic Schematic diagram of the Class D PA

In order to increase the output level of a Class D PA, bridge topologies are adopted. There are mainly two bridge topologies in Class D PA circuit - half-bridge and full-bridge. Each one has its own advantages. Usually, half-bridge circuit employs two output devices. It's obviously simple and has more flexibility. Full-bridge circuit often needs four output devices. In this topology, each output device only needs to reach half the voltage as a half-bridge amplifier of the same power. But the full-bridge structure is more complicated $[3,4,5]$. Figures 2 and 3 show both topologies conceptually, many detail components are not shown.

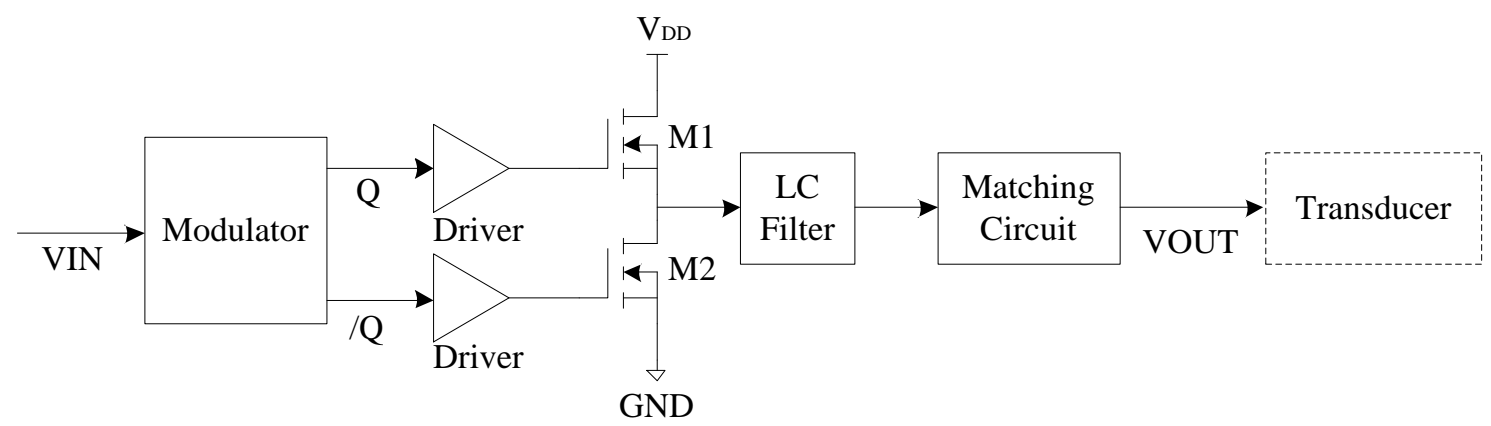

Fig.2 Half-Bridge Class D PA Topology

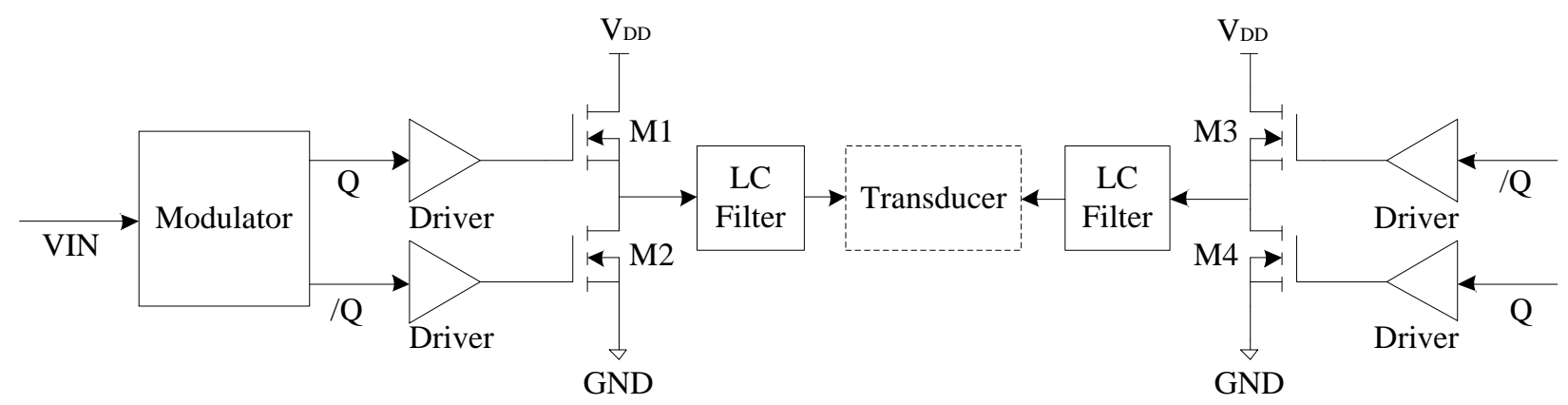

Fig.3 Full-Bridge Class D PA Topology

\section{Design and Implementation}

In this paper, a Class D PA has been designed due to its simple and compact framework as well as ease of implementation. This Class D PA is embedded in a compact transmitter using in a sonar system. When we get down to handling the project, we focus on the specifications of operating frequency, bandwidth and output power, which are crucial for the performance of a sonar system. Figure 4 shows the block diagram of the designed Class D PA.

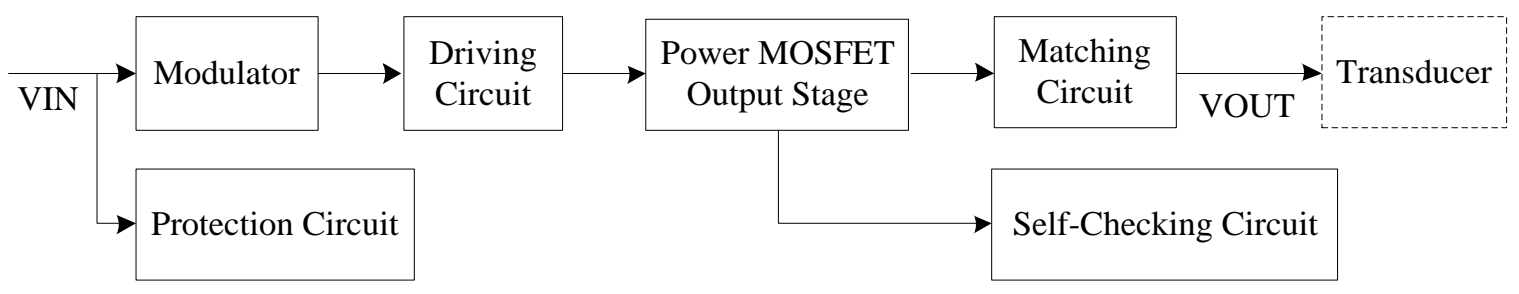

Fig.4 Block Diagram of the Designed Class D PA 
In our design, Half-bridge topology is adopted to increase the output level of the amplifier, as well as to simplify the circuitry. There are modulator, driving circuit, power MOSFET output stage, matching circuit, self-checking circuit and protection circuit in the scheme.

When the $V_{\text {IN }}$ signals from preceding stage is coming, they were first isolated by magnetic couplers. A modulator is designed to generate CW and FM signals for the driving circuit.

The driving circuit uses IRS2110S from International Rectifier as the main driver chip. The IRS2110S are high voltage, high speed power MOSFET and IGBT drivers with independent high-side and low-side referenced output channels. Proprietary HVIC and latch immune CMOS technologies enable ruggedized monolithic construction [6]. For the use of push-pull transformer in the following stage, the driver chip is set to a double low-side driver mode. The inputs of IRS2110S were Q and /Q, Q to the HIN pin and /Q to the LIN pin. The outputs - HO and LO are then sent to the gate pins of the following power MOSFETs. The input of the MOSFET is usually square wave signal. The transistors operate in a switch condition - periodically on and off according to the input signal [7].

Two MOSFETs are employed in a half-bridge topology. One MOSFET gets Q at the gate pin, and the other gets /Q. In the first half period of the emission signal, the $Q$ keeps at a high voltage level while the /Q must be low to ensure the bottom MOSFET is shut down. In the last half period of the emission signal, the Q must be low while the /Q keeps at a high voltage level. This can ensure that at any time, the upper MOSFET and the bottom MOSFET are not working simultaneously.

The high power output signals from the MOSFETs are then sent to the matching circuit (included the LC filter in it). The matching circuit is actually a two order Butterworth filter. The Butterworth filter has good performances in fading characteristic and phase characteristic. More fortunately, there are also no strict requirements when choosing components. Simultaneously, a self-checking circuit is built to indicate the working state of the amplifier.

Considering there are large power signals flowing through the PA, we supply protection circuit to ensure people and equipment safety in the work. The protection circuit has two basic functions:

(1) To ensure the PA won't work too long in an emitting period;

(2) To ensure the driver signals Q and /Q won't be both at high voltage level at any time.

When designing a PA, the power supply system is also very important. Figure 5 shows the block diagram of our power supply system.

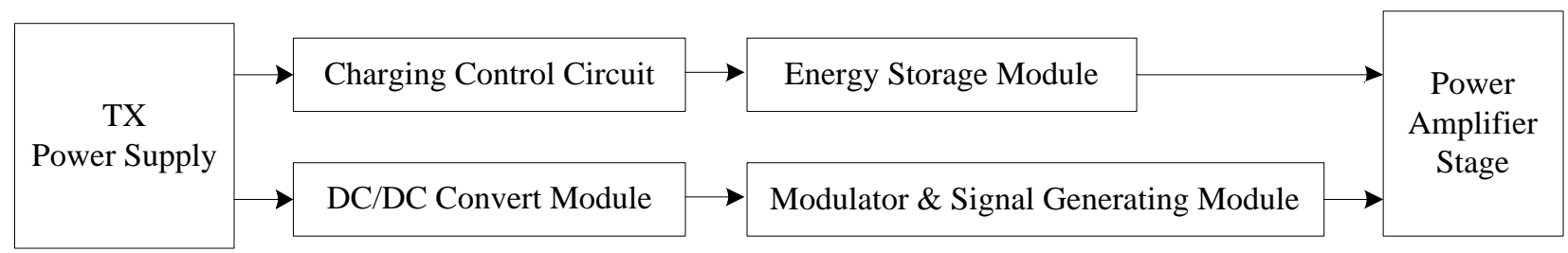

Fig.5 Block Diagram of the Power Supply System

The TX power supply provides a $48 \mathrm{~V}$ voltage for the PA. On the one hand, the $48 \mathrm{~V}$ goes to the charging control circuit and then the energy storage module. The energy storage module is used to provide durable large current when the power amplifier emits signals over a long time. On the other hand, $48 \mathrm{~V}$ goes to the DC/DC convert module. The DC/DC convert module converts $48 \mathrm{~V}$ to lower levels of $15 \mathrm{~V}$ and $5 \mathrm{~V}$ for the modulator and signal generating module.

\section{Measurement Results}

A prototype Class D PA has been fabricated based on a transmitter platform used in a sonar system. The Class D PA occupies a PCB area of $160 \mathrm{~cm}^{2}$ including connectors. For a high-powered audio amplifier a big cooling board is necessary to keep the electronics relatively cool. One connector together with cables is used to connect the PA PCB to the signal generator, the oscilloscope and the stabilized voltage supply.

The measurement results of the Class D PA are shown in Table 1. 
Table 1. Measurement Results of Class D PA

\begin{tabular}{|c|c|c|c|}
\hline Specification & Unit & Sample \#1 & Sample \#2 \\
\hline Low Power Supply 1 & {$[\mathrm{V}]$} & 14.44 & 14.43 \\
\hline Low Power Supply 2 & {$[\mathrm{V}]$} & 4.97 & 4.97 \\
\hline High Power Supply & {$[\mathrm{V}]$} & 48 & 48 \\
\hline Pulse Width Protection Time & {$[\mathrm{ms}]$} & 17 & 18 \\
\hline Center frequency & {$[\mathrm{kHz}]$} & 100 & 100 \\
\hline Bandwidth & {$[\mathrm{kHz}]$} & 5.2 & 5.4 \\
\hline Output Power & {$[\mathrm{W}]$} & 800 & 766 \\
\hline
\end{tabular}

\section{Summary}

A $100 \mathrm{kHz}$ half-bridge switched Class D power amplifier embedded in a sonar equipment has been designed, fabricated and measured. Measurements were carried out under laboratory conditions. Prototypes, proposed in this paper, demonstrate a $760 \mathrm{~W}+$ output power and about $5 \mathrm{kHz}$ bandwidth while operating on a center frequency of $100 \mathrm{kHz}$. The proposed design shows higher output power and higher stability.

\section{References}

[1] Tian Tan: Sonar technology (Second Edition, Harbin Engineering University press, Harbin 2010).

[2] Eric Gaalaas: Class D Audio Amplifiers: What, Why, and How, Analog Dialogue 40-06, June (2006).

[3] Rod Elliott: Class D Audio Amplifiers - Theory and Design (2005).

[4] Liwei Yang, Limei Xu: Class D Power Amplifier for Audio Beam System, Proceedings of the 2007 IEEE, International Conference on Mechatronics and Automation, August 5-8, 2007, Harbin, China.

[5] Tsai-Pi Hung, Jeremy Rode, Lawrence E. Larson and Peter M. Asbeck: Design of H-Bridge Class-D Power Amplifiers for Digital Pulse Modulation Transmitters, IEEE Transactions on Microwave Theory and Techniques, Vol. 55, No. 12, December 2007.

[6] International Rectifier: IRS2110 HIGH AND LOW SIDE DRIVER, Data Sheet No. PD60249.

[7] International Rectifier: Class D Audio Amplifier Basics, Application Note AN-1071. 